\title{
Partial Parameters of Random Network Models for Internet of Things
}

\author{
Jing $\mathrm{Su}^{1}$, Fei Ma ${ }^{1}$, Bing Yao ${ }^{1,}$, Hui Sun ${ }^{1}$, Xiaohui Zhang ${ }^{1}$, Ming Yao ${ }^{2, b}$ \\ ${ }^{1}$ College of Mathematics and Statistics, Northwest Normal University, Lanzhou, 730070 CHINA \\ ${ }^{2}$ Department of Information Process and Control Engineering, Lanzhou Petrochemical College of \\ Vocational Technology Lanzhou, 730060 CHINA
}

aemail: yybb918@163.com, bemail:yybm918@163.com

Keywords: Scale-free; Dynamic Equation; Cumulative Distribution; Algorithm.

\begin{abstract}
A logistical network is related with traffic networks, information networks, multiple goods networks in general. In the view of mathematics, a logistics is the compound result of three or more other networks. We design random multiple models by network operations based on stable transport networks, and measure dynamic differential equations, degree distribution, cumulative distribution of the models.
\end{abstract}

\section{Introduction and Concepts}

In study of logistics, we can apply network operations to build up some models to simulate real situations of the world. It seems to be difficult using simpler vertices and edges to form a model that can imitate the interflow of goods and materials, since most of logistical networks are very complex. Very often, a logistical network is related with weighted traffic networks, changed information networks, multiple goods networks, and so on. In other words, a logistics is the compound result of three or more other networks. We may be facing various connections between networks. In a stable period, a transport (road/railway/airlane) network is not changed, so we can use (diracted/weighted) graphs of graph theory to describe a transport network. But two cities are complex, there are many goods that will be transformed from one to another one. The information about these goods form networks, in detail, the factories and retailers as well as buyers on these goods construct some networks. So, the typical graphs of graph theory are not suitable to deal with such complicated situations.

Another example, a social network (Social Network Service, SNS) contains many small social groups/parties, and there are connections between these social groups. Network researchers want to study and imitate social networks, even measure their efficiency. Again, people make machine equipment, or in detail, many groups of particular men produce many groups of different machines. A biological network (gene network) very often contains many motifs.

We design random multiple models for dealing with the above problems in this article, and propose particular models by means of network operations based on stable transport networks. We measure dynamic differential equations, degree distribution, cumulative distribution to the models for the effectiveness of the models.

A. Introduction

In researching networks one have to consider two processes:Many-to-One, several models $N_{1}(t), N_{2}(t), \ldots, N_{\mathrm{m}}(t)$ can be composed into a large model $N(t)$. Conversely, One-to-Many, one can decompose a large model $N(t)$ into smaller models $N_{1}(t), N_{2}(t), \ldots, N_{\mathrm{m}}(t)$. We want to transfer some characteristics of the models $N_{1}(t), N_{2}(t), \ldots, N_{\mathrm{m}}(t)$ into $N(t)$ in the process Many-to-One, and vice versa in the process One-to-Many (Ref. [7], [10], [13], [14]).

B. Concepts

We, first, introduce definitions, terminology and notation used in this article. A network model $N(t)$ is a mathematical and dynamic model according to time $t$ for the purpose of understanding and describing real networks. In the network model $N(t)$ having vertex set $V(t)$ and edge set $E(t)$, an operation vertex-set is a subset $V^{*}(t)$ of $V(t)$ (see Fig. 1), an operation edge-set is a subset $E^{*}(t)$ of $E(t)$. For example, $V^{*}(t)$ is the set of dominating vertices of $N(t), E^{*}(t)$ is the set of some matching 
edges of $N(t)$. In scale-free networks, one focus on those hub vertices or large-degree vertices of the networks. In the following discussion, let $L_{i}(t)$ be a network model having vertex set $V_{i}(t)$ and edge set $E_{i}(t)$ with integers $i \geq 1$ at time step $t, V^{*}{ }_{i}(t)$ and $E^{*}{ }_{i}(t)$ are the operation vertex-set and the operation edge-set of $L_{i}(t)$.

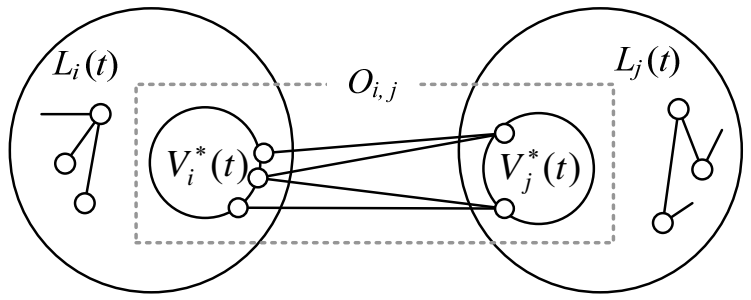

Figure1. A 2-operator $O_{i, j}$ on two operation vertex-sets $V^{*}{ }_{i}(t)$ and $V^{*}{ }_{j}(t)$.
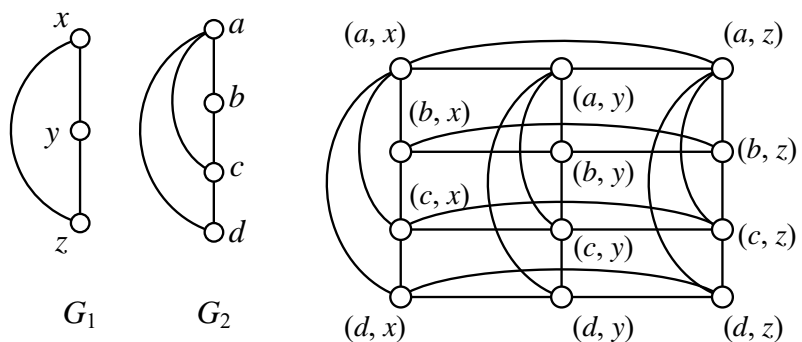

Figure2. A Cartesian product $G_{1} \diamond G_{2}$ of two graphs $G_{1}$ and $G_{2}$.

In [8], the authors proposed: An $m$-dimension $n$-rank mixed-network model $N(t)$ is defined as $N(t)=N(L(t), O(t))$ with integers $m, n \geq 2$, where $L(t)=\left\{L_{1}(t), L_{2}(t), \ldots, L_{\mathrm{m}}(t)\right\}$ is a group of subnetwork models, $O(t)=\left\{O_{1}(t), O_{2}(t), \ldots, O_{\mathrm{n}}(t)\right\}$ is the operation group with $\mathrm{t} \in[a, b] . N(t)$ is obtained by doing each operation of the operation group $O$ on all subnetwork models of $L(t)$.
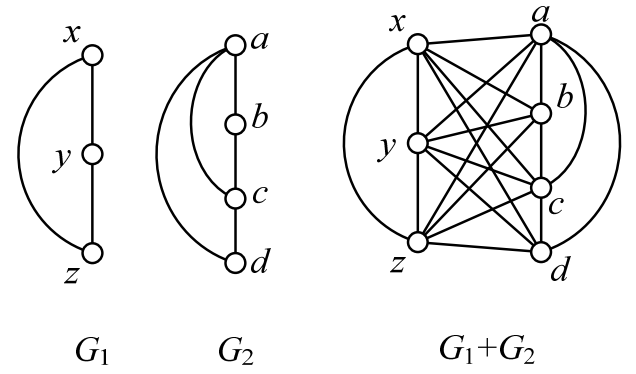

$G_{1}+G_{2}$
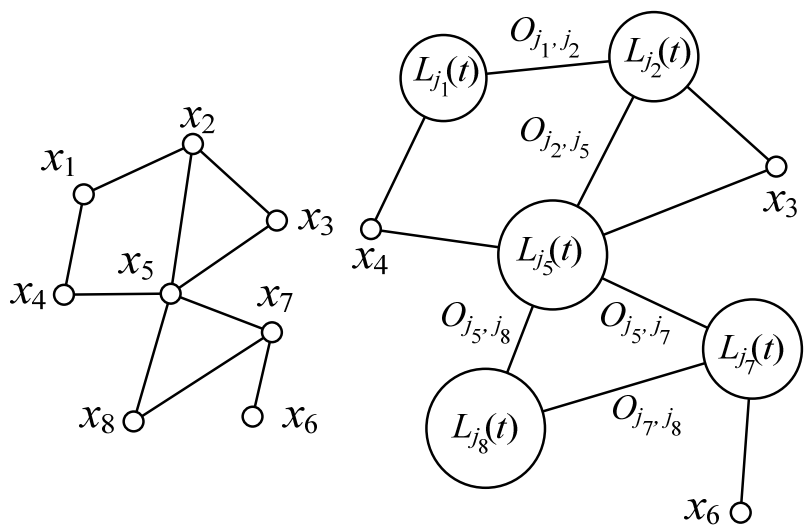

Figure4. Left is the base $H$, right is a multiple network model $H(L, O)(t)$, but some vertices $x_{3}, x_{4}, x_{6}$ of the base $H$ are not substituted by generators of the generator set $L(t)$.

We call each model $L_{i}(t)$ a generator. A 2-operator $O_{i, j}$ (see Fig.1) is a network operation on two generators, for example, the Cartesian product (see Fig.2) is a 2-operator, the join (see Fig.3) is a 2 -operator too. Especially, "joining a vertex (or generator) with another vertex (or generator) by an edge" is regarded as a 2-operator on them. We write $n_{v}^{j}(t) \geq 2$ and $n_{e}^{j}(t) \geq 1$ be the numbers of vertices and edges of the $j$ th generator $L_{i}(t)$ of $L(t)$, respectively, and require that each generator of $L(t)$ to be connected; any two generators of $L(t)$ can be operated by a certain 2-operator $O_{i, j}$ of the 2 -operator set $O$, here after. We are ready to present the concept of multiple network models that differs from one shown in [8] we define

Definition1. (Multiple network model) Suppose that $L(t)$ is a set of generators $L_{1}(t), L_{2}(t), \ldots$, $L_{\mathrm{n}}(t)$ with $t \in[a, b] ; O=\left\{O_{\mathrm{i}, j}: \sim 1 \leq i, j \leq m\right\}$ is a 2-operator set with integers $m, n \geq 1 ; H$ is a simple and connected graph having $n_{v}{ }^{*}$ vertices, called a base. A multiple network model, denoted as $H(L, O)(t)$, is constructed by letting some vertices of $H$ stand for generators $L_{i}(t)$ in $L(t)$, each edge $x_{i} x_{j}$ of $H$ to indicates a 2-operator $O_{i, j}$ on two generators $L_{i}(t)$ and $L_{j}(t)$.

Each 2-operator $O_{i, j}$ does not change with time $t$, so it differs from the operator $O_{i}(t)$ in [8]. The number of the 2-operators $O_{i, j}$ used in $H(L, O)(t)$ does not exceed the cardinality $|E(H)|$ of edge set $E(H)$ of the base $H$, and each generator $L_{i}(t)$ of $H(L, O)(t)$ (or vertices of $H$ ) has been operated with other $\operatorname{deg}_{H}\left(x_{i}\right)$ generators, where $\operatorname{deg}_{H}(x i)$ is the degree of vertex $x_{i}$ of $H$. Notice that there are many multiple network models $H(L, O)(t)$, since each vertex $x_{i}$ indicates a generator $L_{i}(t)$ at random. So, we can define a base-model space $S(L, O ; H)=\left\{H(L, O)(t): L_{i}(t) \in L(t), O_{i, j} \in O\right\}$ on the base $H$, the 
generator set $L(t)$, the operator set $O$ and the operation relationship $H$. Here, we allow some extreme situations happen. For example, $H$ is an element of $S(L, O ; H)$ in which every generator $L_{i}(t)$ contracts/shrinks into a vertex; or some part of vertices of $H$ are regarded as the trivial graphs having one vertex and no edge in $H(L, O)(t)$. (see Fig.4) Thereby, if $n_{v}^{*} \leq|L(t)|$ and it allows duplication, we have $n+1$ elements in $L(t) \cup\{\phi\}$, and can take $n_{v}{ }^{*}$ elements from $L(t) \cup\{\phi\}$ for arranging them to the vertices of $H$ in order to produce $(n+1)^{n v^{*}}$ multiple network models $H(L, O)(t)$; if $n_{v}{ }^{*}>|L(t)|$, we may have $2 \cdot 2 \cdot \ldots \cdot 2=2^{n v^{*}}$ multiple network models $H(L, O)(t)$.

Theorem1. The base-model space $S(L, O ; H)$ contains $(n+1)^{n v^{*}}$ multiple network models if $n_{v}{ }^{*} \leq|L(t)|$, and otherwise $2^{n v^{*}}$ multiple network models if $n_{v}{ }^{*}>|L(t)|$.

We call a model of the base-model space $S(L, O ; H)$ a planar multiple network model if $H$ is a planar graph (Ref. [15]), a $r$-partite multiple network model if $H$ is a $r$-partite graph. A 2-partite multiple network model can be use to imitating: Many groups of particular men produce many groups of different machines. $H$ is a base defined in Definition1. Suppose that $v_{i, j}(t)$ and $e_{i, j}(t)$ are the numbers of new added vertices and edges when doing the operator $O_{i, j}$ on two generators $L_{i}(t)$ and $L_{j}(t)$ of $H(L, O)(t)$. We can divide the vertex set $V(H)$ of the base $H$ into two parts $X$ and $Y$ such that each vertex of $X$ does not correspond a generator, but each vertex of $Y$ corresponds a generator in $H(L, O)(t)$. Let $E^{*}$ be the edge subset of $H$ such that two ends of each edge of $E^{*}$ correspond two generators. We have the numbers of vertices and edges of $H(L, O)(t)$ as follows

$$
n_{v}(t)=|X|+\sum_{y, y, E E} v_{i, j}(t)+\sum_{j=1}^{n} c_{j} n_{v}^{j}(t), n_{e}(t)=\left|E(H) \backslash E^{*}\right|+\sum_{y, y, E} e_{i, j}(t)+\sum_{j=1}^{n} c_{j} n_{v}^{j}(t)
$$

where coefficients $c j$ with $j=1,2, \ldots, n$ are non-negative integers, and at least one $c_{j,} \geq 1$ for some $j_{r}$; and $c_{i}=0$ means that the $i$ thenerator $L_{i}(t)$ does not appear in $H(L, O)(t), c_{i} \geq 1$ indicates the number of $L_{j}(t)$ appeared in $H(L, O)(t)$; $\sum_{j=1}^{n} c_{j}$ is equal to the number $n_{v}^{*}$ of vertices of the base $H$; the partial average degree of the $j$ th generator $L j(t)$ is $\langle k\rangle_{j}=2 n_{e}^{j}(t) / n_{v}^{j}(t),\langle\bar{k}\rangle=\frac{1}{n_{v}^{*}} \sum_{j=1}^{n} c_{j}\langle k\rangle_{j}$ is the mean-average degree, and $\bar{n}_{v}(t)=\sum_{j=1}^{n} c_{j} n_{v}^{j}(t) / n_{v}^{*}, \bar{n}_{e}(t)=\sum_{j=1}^{n} c_{j} n_{e}^{j}(t) / n_{v}^{*}$ givethe main average degree $\langle k\rangle^{*}=\frac{2 \bar{n}_{e}(t)}{\bar{n}_{(}(t)}$.

\section{Partial topological parameters of multiple models}

One can compute the average degree of a network model by the degree spectrum of the model. However, it is not easy to realize such aim for the multiple network models of the base-model space $S(L, O ; H)$. Therefore, we consider some uniformly cases in the following discussion.

First, we write $N(t)=H(L, O)(t)$ as a multiple network model defined in Definition1, so $N(t)$ has its own vertex number and edge number are $n_{v}(t)=|V(t)|$ and $n_{e}(t)=|E(t)|$, respectively. Suppose that each vertex $x_{i}$ of $H$ corresponds a generator $L_{j .}$ of $N(t)$ with $t \in[a, b]$. (see Fig.5)
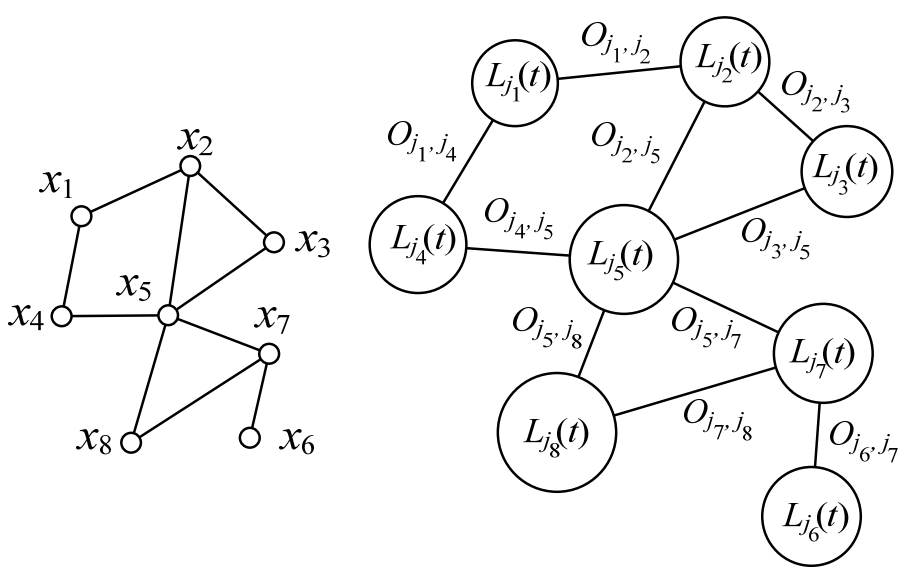

Figure5. Left is the base $H$, right is a multiple network model $H(L, O)(t)$, since each vertex $x i$ of $H$ corresponds to a generator

$L_{j i}(t)$ of $H(L, O)(t)$ with $i=1,2, \ldots, 8$; each edge $x_{r} x_{S}$ of $H$ corresponds a 2-operator $O_{j r j s}$ on two generators $L_{j r}(t)$ and $L_{j s}(t)$. It is allowed that two generators $L_{j r}(t)$ and $L_{j s}(t)$ are isomorphic mutually.

Second, we say that a base-model space is closed to a property if each generator $L_{i}(t)$ of $L(t)$ 
holds the property such that every element of the base-model space has the property too.

A. Average degree

Since $|X|=0$ and $\left|E(H) \backslash E^{*}\right|=0$ (also, $E^{*}=E(H)$ ) in Eq.(1) by the above contract, so we can see: each vertex $x_{i}$ of $H$ corresponds a generator $L_{j i}(t)$ of $N(t)$, thus, Eq. (1) becomes

$$
n_{v}(t)=\sum_{x, x \in E(H)} v_{i, j}(t)+\sum_{j=1}^{n} c_{j} n_{v}^{j}(t), n_{e}(t)=\sum_{x, x \in E(H)} e_{i, j}(t)+\sum_{j=1}^{n} c_{j} n_{e}^{j}(t)
$$

We, by Eq. (2), have

$$
2 \sum_{j=1}^{n} c_{j} j_{e}^{j}(t)=\sum_{j=1}^{n}\langle k\rangle_{j} c_{j} n_{v}^{j}(t) \sim\langle\bar{k}\rangle \sum_{j=1}^{n} c_{j} n_{v}^{j}(t)
$$

and moreover $2\left[n_{e}(t)-\sum_{x, y \in E(H)} e_{i, j}(t)\right] \sim\langle\bar{k}\rangle \sum_{j=1}^{n} c_{j} n_{v}^{j}(t)$ and $2\left[n_{v}(t)-\sum_{x, x \in E(H)} v_{i, j}(t)\right]=\sum_{j=1}^{n} c_{j} n_{v}^{j}(t)$. which show that the average degree $\langle k\rangle$ of $N(t)$ is approximate to the main average degree $\langle\bar{k}\rangle$ for smaller values $\sum_{x, x \in E(H)} e_{i, j}(t)$ and $\sum_{x, y \in E(H)} v_{i, j}(t)$. Similarly with the form (3), we have $2 \bar{n}_{e}(t)=\frac{2}{n_{v}^{*}} \sum_{j=1}^{n} c_{j} n_{e}^{j}(t) \sim \frac{\langle\bar{k}\rangle}{n_{v}^{*}} \sum_{j=1}^{n} c_{j} n_{v}^{j}(t)$, which means the main average degree

$$
\langle k\rangle^{*}=\frac{2 \bar{n}_{e}(t)}{\bar{n}_{v}(t)} \sim\langle\bar{k}\rangle
$$

Therefore, $\langle k\rangle \sim\langle k\rangle^{*}$, this result holds true for Eq. (1) too. The above facts give us a result about sparseness as follows

Theorem2. The base-model space $S(L, O ; H)$ is closed to the sparseness if each generator $L_{j}(t)$ is sparse in $N(t)$.

\section{B. Velocity}

According to the definition of velocity of a network model introduced in [9], the velocity of $N(t)$ is defined as

$$
V_{e l}(N(t))=\frac{\partial n_{v}(t)}{\partial t} \cdot \frac{\partial n_{e}(t)}{\partial t}
$$

It seems to be difficult to compute the exact value of the velocity $\operatorname{Vel}(N(t))$ by Eq. (2), even it regards that all of $v_{i, j}(t)$ and $e_{i, j}(t)$ in Eq. (2) are equal to zero. Notice that $N(t)$ is assembled by the generators though 2-operators, but growing by itself, in other words, the each generator $L_{j}(t)$ is independent of other remainder generators in $N(t)$. By the above reason of independence, we can define the mean-velocity $\overline{\operatorname{Vel}}(N(t))$ of $N(t)$ as follows

$$
\overline{\operatorname{Vel}}(N(t))=\frac{1}{n_{v}^{*}} \sum_{j=1}^{n} c_{j} \partial_{j} \overline{\operatorname{Vel}}(N(t))=\frac{1}{n_{v}^{*}} \sum_{j=1}^{n} c_{j} \frac{\partial n_{v}^{j}(t)}{\partial t} \cdot \frac{\partial n_{e}^{j}(t)}{\partial t}
$$

where $\partial_{j} \overline{\operatorname{Vel}}(N(t))=\operatorname{Vel}\left(L_{j}(t)\right)=\frac{\partial n_{v}^{j}(t)}{\partial t} \cdot \frac{\partial n_{e}^{j}(t)}{\partial t}$ is called the partial velocity of the $j$ th generator $L_{j}(t)$, coefficients $c_{j} \geq 0$ are defined in Eq. (1).

Example1. The BA-model (ref. [2]) has a constant velocity. Clearly, the mean-velocity $\overline{V e l}(N(t))$ will be a constant if each velocity $\operatorname{Vel}\left(L_{j}(t)\right)$ in the formula (6) is a constant with $j=1,2, \ldots, n$. This is an example of the closeness of $N(t)$. Obviously, the velocity of a model is not related with the topological structure of the model.

Example2. If each pair of vertex number $n_{v}^{j}(t)$ and edge number $n_{e}^{j}(t)$ holds $n_{v}^{j}(t) \sim O\left(n_{v}^{j}(t) \ln \left[n_{v}^{j}(t)\right]\right)$ for $j=1,2, \ldots, n$, we get

$$
\overline{\operatorname{Vel}}(N(t))=\frac{1}{n_{v}^{*}} \sum_{j=1}^{n} c_{j} \partial_{j} \overline{\operatorname{Vel}}(N(t))=\frac{1}{n_{v}^{*}} \sum_{j=1}^{n} c_{j}\left[\frac{\partial n_{v}^{j}(t)}{\partial t}\right]^{2} \ln \left(\frac{n_{v}^{j}(t)}{e}\right)
$$

Example3. If the vertex number $n_{v}^{j}(t)$ and edge number $n_{e}^{j}(t)$ of the $j$ th generator $L_{j}(t)$ holds $\frac{\partial n_{e}^{j}(t)}{\partial t} \sim A_{j}\langle k\rangle_{j} \frac{\partial n_{v}^{j}(t)}{\partial t}$ for $j=1,2, \ldots, n$, where $\langle k\rangle_{j}$ is the average degree of the $j$ th generator $L_{j}(t)$ and $A_{j}$ is a constant, we then have the mean-velocity

$$
\overline{\operatorname{Vel}}(N(t))=\frac{1}{n_{v}^{*}} \sum_{j=1}^{n} c_{j} \operatorname{Vel}\left(L_{j}(t)\right)=\frac{1}{n_{v}^{*}} \sum_{j=1}^{n} c_{j} A_{j}\langle k\rangle_{j}\left[\frac{\partial n_{v}^{j}(t)}{\partial t}\right]^{2}
$$




\section{Diameter}

Let $D^{*}, D(H)$ and $D\left(L_{i}(t)\right)$ be the diameters of the multiple network model $N(t)$, the base $H$ and the $j$ th generator $L_{j}(t)$ with $j=1,2, \ldots, n$, respectively. Then each shortest path $P\left(x_{i, s}, x_{j, r}\right)$ of $N(t)$ has the length less than $D\left(L_{i}(t)\right)+D(H)+D\left(L_{j}(t)\right)$, where the vertex $x_{i, s} \in V\left(L_{i}(t)\right)$ and $x_{j, r} \in V\left(L_{j}(t)\right)$ at time step $t$. So, we have

Theorem3. If each generator $L_{j}(t)$ in a multiple network model $H(L, O)(t)$ is small-world, so is $H(L, O)(t)$, and then the diameter $D^{*}$ of $H(L, O)(t)$ holds $D^{*} \leq D(H)+2 \max \left\{D\left(L_{i}(t)\right): 1 \leq \mathrm{i} \leq n\right\}$.

The above Theorem3 implies the base-model space $S(L, O ; H)$ is closed to the small-world property. Bolloba's and Riordan [3] have proven that the diameter $\operatorname{diam}\left(G_{m}^{n}\right)$ of a scale-free graph $G^{n}{ }_{m}$ on $n$ vertices holds

$$
\frac{(1-\varepsilon) \log n}{\log \log n} \leq \operatorname{diam}\left(G_{m}^{n}\right) \leq \frac{(1+\varepsilon) \log n}{\log \log n}
$$

\section{Partial dynamic differential equations and degree distributions}

The dynamic differential equations $\frac{\partial_{i} k_{i}(t)}{\partial t}=f\left(t, k_{i}(t), \Pi_{i}\right)$ (also, continuum equations introduced in [5],[6]) and degree distributions $P(k)$ are the important topological properties of scale-free networks in literature. However, it is not slight and easy to show the scale-free behavior of many networks, due to they are random and complex.

1) Partial degree distributions: Notice that $N(t)$ is assembled by the generators $L_{j}(t)$ that are mutually independent of each other in $N(t)$. Thereby, we can define the main degree distribution $P_{\text {main }}(k)$ of $N(t)$ as

$$
P_{\text {main }}(k)=\frac{1}{n_{v}^{*}} \sum_{j=1}^{n} c_{j} \partial_{j} P(k)
$$

where $\partial_{j} P(k)$ is the degree distribution of $L_{j}(t)$, we call it the $j$ th partial degree distribution of $N(t)$.

If each partial degree distribution $\partial_{j} P(k) \sim k^{-\gamma_{1}}$ with $\gamma_{j}>0$ and $j=1,2, \ldots, n$, we then define the main degree exponential $\bar{\gamma}=\frac{1}{n_{v}^{*}} \sum_{j=1}^{n} c_{j} \gamma_{j}$. Furthermore, by Eq. (10), we can estimate $P_{\operatorname{main}}(k) \sim \sum_{j=1}^{n} \frac{c_{j} k^{-\gamma_{j}}}{n_{v}^{*}} \sim \sum_{j=1}^{n} \frac{c_{j} k^{-\bar{\gamma}}}{n_{v}^{*}}=k^{-\bar{\gamma}}$ When $2<\gamma_{j}<3$ for $j=1,2, \ldots, n$, thus, $2<\bar{\gamma}<3$. The above deduction proves a result

Theorem4. The base-model space $S(L, O ; H)$ is closed to the scale-freeness if each generator $L_{j}(t)$ holds $\partial_{j} P(k) \sim k^{-\gamma, j}$ with $2<\gamma_{j}<3$ in $N(t)$.

2) Partial dynamic differential equations: For $j=1,2, \ldots, n$, suppose that the partial dynamic differential equation of the $j$ th generator $L_{j}(t)$ in $N(t)$ is as

$\frac{\partial_{j} k_{i}(t)}{\partial t}=\frac{\partial k_{i, j}(t)}{\partial t}=r_{j}(t) k_{i, j}(t)+s_{j}(t)$

where $k_{i, j}(t)$ is the degree of a randomly selected vertex $i$ of $L_{j}(t)$. Furthermore, we define the mean-degree $\overline{k_{i}(t)}$ of $N(t)$ by $\overline{k_{i}(t)}=\sum_{j=1}^{n} \frac{c_{j}}{n_{v}} k_{i, j}(t)$ and the dynamic mean-equation

$$
\frac{\partial \bar{k}_{i}(t)}{\partial t}=\sum_{j=1}^{n} \frac{c_{j}}{n_{v}^{*}} \frac{\partial_{j} k_{i}(t)}{\partial t}=\sum_{j=1}^{n} \frac{c_{j}}{n_{v}^{*}} \frac{\partial k_{i, j}(t)}{\partial t}=\sum_{j=1}^{n} \frac{c_{j}}{n_{v}^{*}}\left[r_{j}(t) k_{i, j}(t)+s_{j}(t)\right]
$$

We can solve Eq. (12) in particular cases.

Example4. Suppose that each generator $L_{j}(t)$ holds a "generalized dynamic BA-equation" (Ref. [11], [12]) of the form

$$
\frac{\partial k_{i, j}(t)}{\partial t}=m_{j}(t) \cdot k_{i, j}(t)
$$

with $j=1,2, \ldots, n$, and $\bar{m}(t)=\sum_{j=1}^{n} m_{j}(t)$ is the growing mean-value, then we have

$$
\frac{\partial \bar{k}_{i}(t)}{\partial t}=\sum_{j=1}^{n} \frac{c_{j}}{n_{v}^{*}} \frac{\partial k_{i, j}(t)}{\partial t}=\sum_{j=1}^{n} \frac{c_{j}}{n_{v}^{*}} m_{j}(t) k_{i, j}(t) \approx \bar{m}(t) \sum_{j=1}^{n} \frac{c_{j}}{n_{v}} k_{i, j}(t)=\bar{m}(t) \cdot \bar{k}_{i}(t)
$$


Under the initial value $\bar{k}_{i}\left(t_{i}\right)=\bar{m}\left(t_{i}\right)$, the complete solution of Eq. (13) is

$$
\overline{k_{i}}(t)=\bar{m}\left(t_{i}\right) e^{\int_{m}^{m}(x) d x} / e^{\int m_{m}(x) d x}
$$

Solving $t_{i}>M(t, k)$ from $k_{i}(t)<k$ by Eq. (15) enables us to compute all partial degree distributions $\partial_{j} P(k)$ of $N(t)$.

Theorem5. If each generator $L_{j}(t)$ is a linearized BA-model (Ref. [11]), that is, $L_{j}(t)$ has its own partial dynamic differential equation in the form

$$
\frac{\partial k_{i, j}(t)}{\partial t}=\frac{a_{j} k_{i, j}(t)+A_{j}}{b_{j} t+B_{j}}
$$

In the exclusive sense, we call Eq.(16) a "dynamic BA-equation", and a "standard dynamic BA-equation" if $A_{j}=0$.

By assuming that add a new vertex which produces $m_{j}$ new edges at each time step $t$, and take the initial value $k_{i, j}\left(t_{i}\right)=m_{j}$, and $L_{j}(0)$ has $m_{j, 0}$ edges. Thereby, the complete solution of Eq. (16) is $k_{i, j}(t)=\frac{a_{j} m_{j}+A_{j}}{a_{i}}\left(\frac{b_{j} t+B_{j}}{b_{i} t_{i}+B_{i}}\right)^{a_{j} b_{i}}-\frac{A_{j}}{a_{j}}$ and $t_{i}>M_{j}(t, k)$ with $M_{j}(t, k)=\frac{b_{j} t+B_{j}}{b_{i}}\left(\frac{a_{j} m_{j}+A_{j}}{a_{j} k+A_{i}}\right)^{b_{j} / a_{i}}-\frac{B_{j}}{b_{i}}$ from $k_{i, j}(t)<k$. Because $\partial_{j} P\left(k_{i, j}(t)<k\right)=1-\partial_{j} P\left(t_{i} \leq M_{j}(t, k)\right)=1-\int_{0}^{m_{i}(t, k)} p_{j}\left(t_{i}\right) d t$ and the density function $p_{j}\left(t_{i}\right)=\frac{1}{m_{j, 0}+t_{i}}$ under the well-distributed distribution, hence, we obtain the jth partial degree distribution of $N(t)$

$$
\partial_{j} P(k)=\frac{\partial\left[\partial_{j} P\left(k_{i, j}(t)<k\right)\right]}{\partial k}=\frac{a_{j}\left(b_{j} t+B_{j}\right)\left(a_{j} m_{j}+A_{j}\right)^{b / a_{i}}}{b_{j}\left(m_{j, 0}+t\right)\left(a_{j} k+A_{j}\right)^{y /}}
$$

with $\gamma_{j}=1+\frac{b_{j}}{a_{j}}$ that has been proved in [5] and [6]. Clearly, the linearized BA-model having $a_{j}=1, A_{j}=B_{j}=0$ and $b_{j}=2$ is the famous BA-model shown in [2].

Now, we obtain the desired dynamic mean-equation of $N(t)$

$$
\frac{\partial \overline{k_{i}}(t)}{\partial t}=\sum_{j=1}^{n} \frac{c_{j}}{n_{v}^{*}} \frac{\partial_{j} k_{i}(t)}{\partial t}=\sum_{j=1}^{n} \frac{c_{j}}{n_{v}^{*}} \frac{a_{j} k_{i, j}(t)+A_{j}}{b_{j} t+B_{j}} \sim R(t) \bar{k}_{i}(t)+M(t)
$$

where two rational functions $R(t)=\frac{1}{n_{v}^{*}} \sum_{j=1}^{n} c_{j} \frac{a_{j}}{b_{j} t+B_{j}}, M(t)=\frac{1}{n_{v}^{*}} \sum_{j=1}^{n} c_{j} \frac{A_{j}}{b_{j} t+B_{j}}$, Solving Eq.(18) induces the main degree distribution of $N(t)$ as follows

$$
P_{\text {main }}(k)=\frac{1}{n_{v}^{*}} \sum_{j=1}^{n} c_{j} \partial_{j} P(k)=\sum_{j=1}^{n} \frac{c_{j}}{n_{v}^{*}} \cdot \frac{a_{j}\left(b_{j} t+B_{j}\right)\left(a_{j} m_{j}+A_{j}\right)^{b^{\prime} / a_{i}}}{b_{j}\left(m_{j, 0}+t\right)\left(a_{j} k+A_{j}\right)^{\gamma_{j}}}
$$

As all $A_{j}=0$ in the equation (19), also Eq. (16) is a standard dynamic BA-equation, we get

$$
P_{\text {main }}(k) \sim \frac{k^{-\bar{\gamma}}}{n_{v}^{*}} \sum_{j=1}^{n} c_{j} \frac{\left(b_{j} t+B_{j}\right)\left(a_{j} m_{j}\right)^{b / a_{j}}}{b_{j}\left(m_{j, 0}+t\right)} \rightarrow \frac{k^{-\bar{\gamma}}}{n_{v}^{*}} \sum_{j=1}^{n} c_{j}\left(a_{j} m_{j}\right)^{b / a_{j}},(t \rightarrow \infty)
$$

with the main degree exponential $\bar{\gamma}=\frac{1}{n_{v}^{*}} \sum_{j=1}^{n} c_{j} \gamma_{j}$

3) Cumulative distribution The cumulative distribution of a network model $X(t)$ is defined as

$$
P_{\text {cum }}(k)=\frac{1}{n_{v}(t)} \sum_{k \gtrless k} N\left(k^{\prime}, t\right) \sim k^{1-\gamma} \text { (Ref. [4]), where } N\left(k^{\prime}, t\right) \text { is the number of vertices of degree } k^{\prime} \text { in } X(t) \text {. }
$$

Amaral et al. used the cumulative distribution

$$
P_{\text {cum }}(k)=\int_{k}^{+\infty} P(x) d x=1-\int_{0}^{k} P(x) d x
$$

for those models in which it is nearly impossible to obtain some functional form for the degree distribution directly because of strong fluctuations (Ref. 1). There are some proof of $P(k)=-\frac{\partial P_{\text {cum }}(k)}{\partial k}$ for particular network models in literature, rather for all network models. We define the main cumulative distribution $P_{c u m}^{\text {min }}(k)$ of $N(t)$ as

$$
P_{\text {cum }}^{\text {min }}(k)=\sum_{j=1}^{n} c_{j} \partial_{j} P_{\text {cum }}(k)
$$

where $\partial_{j} P_{\text {cum }}(k)=\frac{1}{n_{v}^{*}} \sum_{k 2 k} N_{j}\left(k^{\prime}, t\right)$ is the partial cumulative distribution of the $j$ th generator $L_{j}(t)$, and $N_{j}\left(k^{\prime}, t\right)$ is the number of vertices of degree $k^{\prime}$ in the $j$ th generator $L_{j}(t)$. 
Example6. If each generator $L_{j}(t)$ of $N(t)$ is a linearized BA-model (Ref. Example5), and by Eq. (19) and the formula (21), we get

$$
P_{\text {cum }}^{\text {main }}(k)=\int_{k}^{+\infty} P_{\text {main }}(k) d k=\sum_{j=1}^{n} \frac{c_{j}}{n_{v}^{*}} \int_{k}^{+\infty} \partial_{j} P(k) d k=\sum_{j=1}^{n} \frac{c_{j}}{n_{v}^{n}} \int_{k}^{+\infty} \frac{a_{j} U_{j}(t)}{\left(a_{j} k+A_{j}\right)^{\gamma_{j}}} d k=\sum_{j=1}^{n} \frac{c_{j}}{n_{v}^{*}} \cdot \frac{U_{j}(t)}{\gamma_{j}-1}\left(a_{j} k+A_{j}\right)^{1-\gamma_{j}}
$$

where $U_{j}(t)=\frac{\left(b_{j} t+B_{j}\right)\left(a_{j} m_{j}+A_{j}\right)^{b / a_{j}}}{b_{j}\left(m_{j, 0}+t\right)}$ Therefore, we have shown $P_{\min }(k)=-\frac{\partial P_{\text {cum }}^{\text {min }}(k)}{\partial k}$ about this multiple model $N(t)$, and $\partial_{j} P_{\text {cum }}(k)=\frac{U_{j}(t)}{n_{v}^{*}\left(\gamma_{j}-1\right)}\left(a_{j} k+A_{j}\right)^{1-\gamma / \gamma}$ with $j=1,2, \ldots, n$.

\section{Conclusion and problems}

In the ear of mass data, people are concerned about efficiency of a network rather than the exact values of various parameters of the network. What is the efficiency of a network model? Furthermore, what is an exact method (resp. an efficient method) of mathematics? Along with these two problems, we have designed several types of network models and hope to apply them in study of self-similar and nested complex networks. In fact, it is difficult to obtain the exact values of some parameters of networks, even impossible, since these networks have vast of vertices and edges, and very randomly.

We have not discuss many important parameters, such as the clustering coefficient to the models, even this parameter is very important for the models. More important topic is about the error and attack tolerance of the models. Our models are very far from reality, only stay on testing and exploring from the mathematical view. On the other hands, we are lack of empirical data and examples to explain the models introduced here. In a scale-free network, the degree distribution $\$ \mathrm{P}(\mathrm{k}) \$$, very often, is not related with the numbers of vertices and edges of the network, however, we can ask for Problem1 below.

Problem1. If a model $N(t)$ has its own degree distribution $P(k)$ or cumulative distribution $P_{\text {cum }}(k)$ obtained in explicit functional forms, does $N(t)$ have its own dynamic differential equation $\frac{\partial k_{i}(t)}{\partial t}=f\left(t, k_{i}(t)\right) ?$

Problem2. If some generators $L_{i}(t)$ of a multiple network model $H(L, O)(t)$ are not scale-free, how about the scale-freeness of $H(L, O)(t)$ ?;

\section{Acknowledgement}

The author, Bing Yao, was supported by the National Natural Science Foundation of China under grants No. 61163054, No. 61363060 and No. 61662066. The author, Ming Yao, was supported by the Special Funds of Finance Department of Gansu Province of China under Grant No. 2014-63.

\section{References}

[1] Amaral, L. A. N., Scala, A., Barthelemy, M. and Stanley, H. E., 2000, Classes of small-world networks, Proc. Nat. Acad. Sci. U.S.A., 97, 11149.

[2] Albert-Laszlo Baraba'si and Reka Albert. Emergence of scaling in random networks. lemph $\{$ Science $\} \backslash$ textbf $\{286\}$ (1999) 509-512.

[3] B. Bolloba's and O. Riordan. The Diameter of a Scale-Free Random Graph. Combinatorica 24:1 (2004), 5-34.

[4] S. N. Dorogovstev, A. V. Goltsev, J. F. F. Mendes. Pseufractal scale-free web. Physiacal review 2002, 65, 066122-066125.

[5] Dorogovtsev, S. N. and Mendes, J. F. F. Evolution of networks. Advances in Physics (2002), 51

(4): 1079-1187. 
[6] Xia Liu, Bing Yao, Wanjia Zhang, Xiang' en Chen, Xinsheng Liu, Ming Yao. Uniformly Bound-Growing Network Models And Their Spanning Trees.The 2014 International Conference on Information and Communications Technologies (ICT2014), 2014, pp35-38

[7] Bing Yao, Jing Su, Fei Ma, Xiaomin Wang, Xiyang Zhao, Ming Yao. Exploring Network Operations For Data and Information Networks. submitted (2016)

[8] Bing Yao, Xiaomin Wang, Jing Su, Fei Ma, Ming Yao, Mingjun Zhang, and Jianmin Xie. Methods And Problems Attempt in Scale-Free Models From Complex Networks. submitted (2016).

[9] Bing Yao, Chao Yang, Ming Yao, Hongyu Wang, Xiang'en Chen, Xiaomin Zhang, Mogang Li. Applied Mechanics and Materials, Vol. 380-384(2013) pp 2034-2037. Bing Yao, Hongyu Wang, Ming Yao, Xiang'en Chen, Chao Yang and Xiaomin Zhang. Third International Conference on Information Science and Technology March 23-25, 2013, 738-743. Bing Yao, Xia Liu, Wan-jia Zhang, Xiang'en Chen, Xiao-min Zhang, Ming Yao, Zheng-xue Zhao. 2013 IEEE International Conference on High Performance Computing and Communications and Embedded and Ubiquitous Computing, 2354-2361. Bing Yao, Xia Liu, Wanjia Zhang, Xiang'en Chen, Ming Yao. Applied Mechanics and Materials, Volumes 513-517, pp2444-2448. Bing Yao, Xia Liu, Wanjia Zhang, Xiang'en Chen, Ming Yao. Proceeding of 2014 IEEE 7th Joint International Information Technology and Artificial Intelligence Conference (ITAIC 2014), pp 450-454.

[10] Bing Yao, Jing Su, Fei Ma, Xiaomin Wang, Hui Sun, Ming Yao. Network Models Made By Dynamic Differential Equations. submitted (2016)

[11] Xiaomin Wang, Bing Yao and Jin Xu. arXiv:1601.06357v1 [cs.SI] 24 Jan 2016. Xiaomin Wang, Bing Yao, Jin Xu. ArXiv 1512.08657v1 (2015).

[12] Xiaomin Wang, Bing Yao, Fei Ma, Jing Su, Xiang-en Chen, Ming Yao. Hierarchical Structure and Particular Spanning Trees of Edge-bound Growing Models. 2015 12th International Conference on Fuzzy Systems and Knowledge Discovery (FSKD'15). 978-1-4673-7681-5,2015, IEEE 476-481.

[13] Xiaomin Wang, Bing Yao, Fei Ma, Xiyang Zhao, Jing Su, Xiang-en Chen, Ming Yao. On Composition and Decomposition of Networks. 2015 8th International Conference on BioMedical Engineering and Informatics (BMEI 2015)783-788.

[14] Zhang Zhongzhi, Zhou Shuigeng, Fang Lujun, Guan Jihong, Zhang Yichao. Maximal planar scale-free Sierpinski networks with small-world effect and power-law strength-degree correlation. EPL (Europhysics Letters), 2007, 79: 38007. 